\title{
DETERMINANTES DA EXPANSÃO DO EMPREGO FORMAL: O QUE EXPLICA O AUMENTO DO TAMANHO MÉDIO DOS ESTABELECIMENTOS?
}

\author{
Carlos Henrique L. Corseuil * \\ RODrigo L. MOURA ${ }^{\dagger}$ \\ LAURO RAMOs ${ }^{\ddagger}$
}

\begin{abstract}
Resumo
Esse trabalho tem o intuito de contribuir para a investigação dos determinantes da aceleração no crescimento do emprego formal na última década. As evidências apresentadas apontam que essa aceleração se deve a uma reversão na tendência do número médio de empregados por estabelecimento, em contraponto a uma tendência de crescimento constante no número de estabelecimentos. Investigações mais detalhadas mostram evidências de que esse resultado não é derivado de mudanças na composição setorial que favoreça setores com maiores escalas de operação, nem um aprofundamento do processo de seleção que penaliza os pequenos estabelecimentos. As evidências apontam que a seleção teria contribuído para uma diminuição do tamanho médio, mas esse efeito foi contrabalançado por um nível de emprego maior nas firmas mais novas.
\end{abstract}

Palavras-chave: Emprego Formal, Tamanho dos Estabelecimentos, RAIS

\begin{abstract}
The main goal of this paper is to analyze the increase of Brazilian formal employment growth rate in the last decade. The evidence suggests that this acceleration is due to a reversal in the trend of the average number of employees per establishment. More detailed investigations show evidence that this result is not derived from changes in industry composition that favors sectors with larger scales of operation, or a deepening of the process of selection, which penalizes the smaller establishments. As a matter of fact the selection component would have contributed to a decrease in the average firm size, but this effect was counterbalanced by a higher initial employment level in the most recent firms cohort.
\end{abstract}

Keywords: Formal Employment, Establishment Size, RAIS

JEL classification: J21

\footnotetext{
*IPEA.E-mail:carlos.corseuil@ipea.gov.br

† IBRE/FGV. E-mail: rodrigo. leandro@fgv.br.

‡ IPEA. E-mail:lauro.ramos@ipea.gov.br.
} 


\section{Introdução}

A evolução do mercado formal ao longo dos anos 90 foi marcada por um processo de queda da participação dos postos de trabalho considerados protegidos - setor formal. Com efeito, os dados da Pesquisa Nacional por Amostra de Domicílios, do IBGE, registraram queda contínua da participação dos trabalhadores com carteira de trabalho assinada ao longo da década (Chahad (2006); Ramos \& Ferreira (2005)). Nota-se, entretanto, que, a partir da virada da década, o país experimentou uma reação forte do mercado formal de trabalho, com elevação contínua da participação dos trabalhadores com carteira assinada e também dos contribuintes para a previdência social. ${ }^{1}$

Assim, o objetivo desse artigo é contribuir para um melhor entendimento do que ocorreu no nível de ocupação no mercado de trabalho formal brasileiro nestes dois períodos. Estudos anteriores trouxeram algumas informações nesse sentido, mas ainda não se tem um diagnóstico consensual. Por um lado, alguns deles procuram identificar algum segmento do mercado de trabalho formal que tenha tido um papel preponderante na recuperação observada na primeira metade da década atual. Neste grupo, Cavaleri (2008) destaca as regiões metropolitanas como um segmento no qual a recuperação do emprego formal aparece de forma destacada. Já outros procuram avaliar a contribuição de elementos de políticas públicas para a recuperação do emprego formal. Entre esses, Simão (2009) analisa os papéis da fiscalização do Ministério do Trabalho e Emprego (MTE), e da desburocratização e redução tributária levada a cabo pelo SIMPLES, usando dados do CAGED.

O presente artigo se aproxima mais do primeiro grupo mencionado acima. Mas dada a escassa evidência empírica para o Brasil, o artigo inclui diversas contribuições. Primeiramente, com uma abordagem que procura inovar nas dimensões a serem investigadas: a evolução do número de estabelecimentos e do número médio de empregados por estabelecimento. Dessa forma, conseguimos identificar que o segundo efeito foi predominante na evolução do emprego formal observada na década atual. Além disso, conseguimos identificar os determinantes do crescimento do tamanho médio das firmas chegando a um resultado não encontrado em nenhum estudo para o Brasil: não apenas as firmas cresceram, mas elas passaram a ter mais empregados no momento de sua criação. Ou seja, conseguimos dar uma direção mais precisa sobre a evolução do emprego formal, não apenas citando se um determinado segmento (no caso de Cavaleri (2008), as regiões metropolitanas) ajuda a explicar o crescimento do emprego formal.

Para realizar todas essas análises, a base de dados utilizada é construída a partir dos microdados da Relação Anual de Informações Sociais (RAIS) do Ministério do Trabalho e Emprego (MTE). ${ }^{2}$ A partir dos dados originais montamos um arquivo de estabelecimentos contendo o número de empregados com vínculo ativo em 31 de dezembro de diversos anos (1995 a 2007), ${ }^{3}$ além de outras características do estabelecimento a serem mencionadas adiante.

\footnotetext{
${ }^{1}$ Tafner (2006), capítulo 5, destaca esse fato entre outros relevantes para o entendimento da evolução do mercado de trabalho brasileiro nesse período.

${ }^{2}$ Trata-se de uma base originada de declarações prestadas por todos os estabelecimentos com CNPJ contendo informações a respeito de todos os vínculos empregatícios registrados nos respectivos estabelecimentos.

${ }^{3}$ Os resultados obtidos para o número de empregados, não necessariamente coincidem com as estatísticas oficiais do MTE. Isso se deve ao tratamento a que submetemos os dados originais. Primeiro, impomos uma restrição de contar apenas uma vez um determinado trabalhador numa
} 
A análise da próxima seção mostra alguns fatos estilizados relacionados à evolução do emprego formal, a saber: (i) o número de empregos formais cresceu tanto na década passada como na atual, com maior elevação para a última, (ii) o número de estabelecimentos cresceu de forma praticamente linear em todo o período, (iii) a variação do tamanho médio das firmas apresenta um formato de "U", sendo positiva de 2003 em diante. Sendo assim, consideramos que quaisquer que sejam os determinantes do crescimento do emprego formal nesse período, o mecanismo deve passar de alguma forma pela determinação do tamanho médio das firmas no setor formal. Esse será portanto o foco do presente artigo.

A seção 3 traz a metodologia para avaliar os determinantes da evolução do emprego formal e os resultados. A metodologia consiste em construção de contrafactuais por meio de decomposições simples. Os resultados apontam para o fato de que, apesar de o crescimento do número de estabelecimentos ser o maior responsável pela geração de emprego formal no período analisado, essa variável não importa para a mudança de tendência comentada no primeiro parágrafo, visto que o número de estabelecimentos cresce a uma taxa praticamente constante no período analisado. Logo, a mudança de tendência na evolução do emprego formal se deve quase que integralmente à evolução do componente relacionado ao aumento do tamanho médio dos estabelecimentos. Nossa primeira preocupação é de mostrar que esse crescimento não se deve a uma realocação entre setores de atividade de forma que os estabelecimentos passassem a se concentrar mais em setores que usam ofator trabalho mais intensivamente.

Na seção 3.1 recorremos à teoria econômica para guiar uma análise mais profunda sobre a mudança detectada no componente relacionado ao tamanho médio das firmas entre a década de 90 (primeiro subperíodo) e a atual década (segundo subperíodo). As hipóteses levantadas pelo arcabouço teórico sugerem a análise de algumas estatísticas da distribuição do tamanho por faixa etária dos estabelecimentos, o que é feito na seção 5. Essa análise procura identificar em que medida a média do tamanho dos estabelecimentos sobreviventes seria afetada por alterações na probabilidade de sobrevivência dos menores estabelecimentos. Por fim, apresentamos um breve sumário dos resultados na última seção.

\section{Análise Preliminar da evolução do emprego formal}

Em uma análise preliminar verificamos alguns fatos estilizados sobre a evolução do emprego formal da década passada para a atual. O Gráfico 1 traz a evolução do emprego formal entre 1995 e 2007. ${ }^{4}$ O crescimento do emprego formal foi de aproximadamente 13,9 milhões de postos de trabalho nesse período, o que implica num ritmo de crescimento médio de 1,16 milhões de postos de trabalho por ano. No entanto, podemos notar a existência de padrões um pouco distintos neste ritmo de expansão do emprego formal, tendo como pano de fundo a aceleração do crescimento ao longo do tempo: entre 1995 e 1999, o crescimento anual médio do emprego formal foi de apenas 358

mesma empresa (ou seja, se houver acúmulo de cargos para um mesmo trabalhador numa mesma empresa, o mesmo será contado mais de uma vez pelo MTE, mas apenas uma única vez por nós). Além disso, filtramos algumas observações com registros inválidos para PIS ou CNPJ.

${ }^{4}$ Como a nossa data de referência é o último dia do ano, na prática o Gráfico mostra a evolução do emprego formal no período que incorpora os anos de 1996 a 2007. 
mil; já entre 1999 e 2003, esse número sobe para 1,13 milhões de postos de trabalho por ano; por fim, entre 2003 e 2007, o crescimento médio anual registrado para o emprego formal foi quase 2 milhões de postos de trabalho. ${ }^{5}$ Vale ressaltar, por motivos a serem mencionados mais adiante, que no primeiro e último quinquênio o crescimento foi a uma taxa praticamente constante.

Procurando entender alguns determinantes desse comportamento diferenciado do emprego formal ao longo dos anos analisados, vale destacar que a variação do emprego formal pode ser entendida como resultante da variação conjunta de dois componentes - número de estabelecimentos formais e tamanho médio dos estabelecimentos formais. A evolução de tais componentes pode ser vista nas Figuras 2 e 3, respectivamente. Nota-se de imediato que a evolução do tamanho médio parece ser a responsável pela variação da taxa de crescimento do emprego formal entre os subperíodos mencionados acima. Enquanto o número de estabelecimentos cresce a taxas praticamente constantes em todo o período, o tamanho médio possui comportamentos bastante distintos nos três subperíodos citados, registrando inclusive reversão do sinal. Essa variável diminui 0,46 postos de trabalho por ano entre 1995 e 1999, permanece praticamente inalterada entre 1999 e $2003,{ }^{6}$ e aumenta 0,29 postos por ano entre 2003 e 2007.

Sendo assim, consideramos a reversão do comportamento do tamanho médio dos estabelecimentos entre 1995-1999 e 2003-2007 como o fato relevante a ser entendido. No restante do artigo, nos concentraremos na análise dos determinantes dessa variável, para os subperíodos mencionados acima.

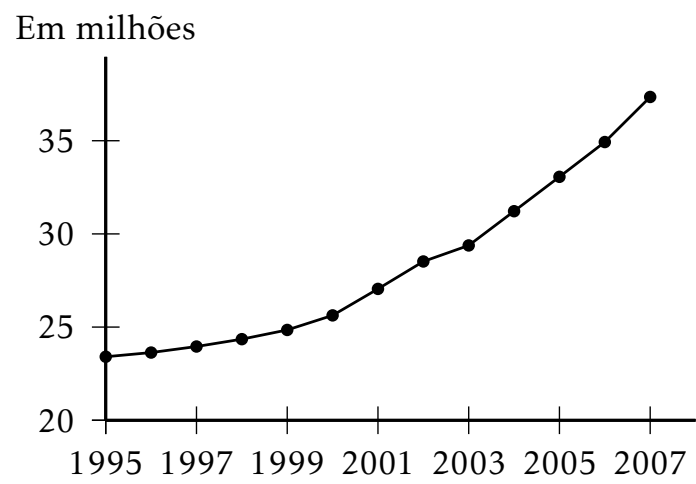

Fonte:Elaboração a partir dos dados da RAIS

Figura 1: Total de empregos formais

\section{Decomposição do emprego e do tamanho das firmas}

Passaremos agora para a análise dos determinantes do tamanho médio dos estabelecimentos numa dimensão ainda mais detalhada. A evolução dessa variável pode ser decomposta em efeito escala e efeito composição, ou seja, quanto é devido a um aumento das contratações formais de fato e quanto é devido simplesmente a uma realocação na composição destas firmas entre,

\footnotetext{
${ }^{5} \mathrm{O}$ crescimento total do emprego formal foi de 1,43 milhões entre 1995 e 1999, 4,5 milhões entre 1999 e 2003, e 7,97 milhões entre 2003 e 2007.

${ }^{6}$ Nesse período a taxa de variação do tamanho médio dos estabelecimentos oscila em torno de uma diminuição média de 0,01 postos de trabalho por ano.
} 


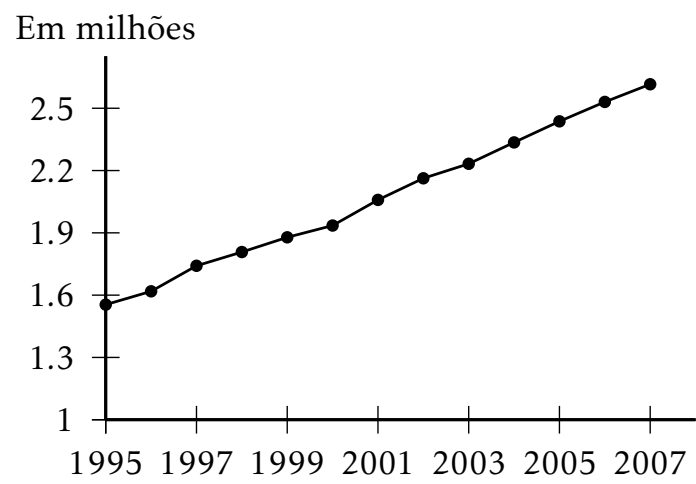

Fonte:Elaboração a partir dos dados da RAIS

Figura 2: Número de estabelecimentos

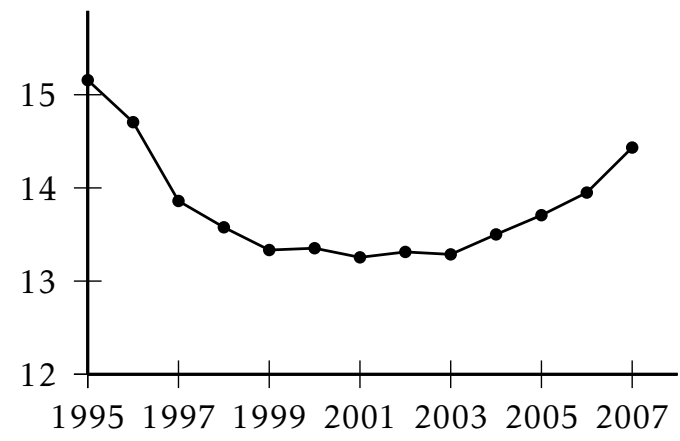

Fonte:Elaboração a partir dos dados da RAIS

Figura 3: Tamanho médio das firmas (número médio de empregados por firma)

por exemplo, setores de atividade. Sabe-se que estabelecimentos industriais tendem a ser maiores do que os de comércio. Sendo assim, uma redistribuição dos estabelecimentos onde se aumenta a concentração da indústria pode induzir um aumento do tamanho médio sem que este aumente em cada um dos grupos considerados.

A Figura 4 abaixo ilustra como todos os efeitos mencionados acima se relacionam com o emprego formal, bem como entre si. Em seguida, descrevemos formalmente essas relações, bem como uma maneira de quantificá-las por intermédio de decomposição simples.

\subsection{Metodologia para quantificar a importância relativa de cada determinante}

A quantificação da importância relativa de cada um dos determinantes na evolução do grau de formalização do mercado de trabalho brasileiro, em cada um dos dois subperíodos considerados, pode ser implementada com o seguinte exercício de decomposição da variação do número de trabalhadores do setor formal entre dois instantes do tempo como:

$$
n_{1} x_{1}-n_{0} x_{0}=n_{\alpha} \Delta x+x_{\alpha} \Delta n
$$




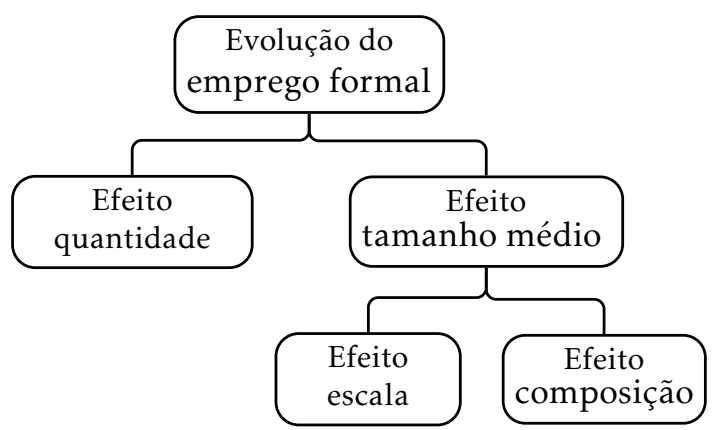

Figura 4: Decomposição da evolução do emprego formal

onde:

$n_{i}$ é o número de firmas no setor formal no instante $i, x_{i}$ é o tamanho (número de trabalhadores) médio das firmas no setor formal no instante $i$, $\Delta n=n_{1}-n_{0}$ e $\Delta x=x_{1}-x_{0}$ são as respectivas variações no número de firmas e no seu tamanho médio entre o início e o final do intervalo de tempo considerado (de 0 a 1, por exemplo, de 1995 a 1999 no caso do primeiro subperíodo), e $n_{\alpha}=\alpha n_{0}+(1-\alpha) n_{1}$ e $x_{\alpha}=(1-\alpha) x_{0}+\alpha x_{1}$ com $\alpha \in[0,1]$ são combinações lineares convexas das respectivas variáveis nos dois instantes de tempo, simetricamente ponderadas. Se $\alpha=1 / 2$ temos, como resultado de uma ponderação idêntica, as médias aritméticas, denotadas abaixo por $n_{m}$ e $x_{m}$. Assim, nesse caso particular:

$$
n_{1} x_{1}-n_{0} x_{0}=n_{m} \Delta x+x_{m} \Delta n=n_{m}\left(x_{1}-x_{0}\right)+x_{m}\left(n_{1}-n_{0}\right)
$$

O primeiro termo à direita da equação (1) pode ser chamado de efeito "tamanho médio" e o segundo de efeito "quantidade" na explicação da variação do total de trabalhadores formais no intervalo considerado. O primeiro componente mostra o seguinte contrafactual: qual teria sido a variação do emprego formal resultante apenas da variação observada no tamanho médio dos estabelecimentos no subperíodo considerado, mantido fixo o número de estabelecimentos no nível médio entre os anos mencionados. O segundo componente, indica qual teria sido a variação do emprego formal resultante apenas da variação observada no número de estabelecimentos no subperíodo considerado, mantendo constante o tamanho dos mesmos no nível médio entre os anos citados.

Caso o primeiro (segundo) componente venha a ser o maior deles, então concluiríamos que o aumento do tamanho médio dos estabelecimentos (o aumento do número de estabelecimentos formais) foi o maior responsável pelo recente aumento do emprego formal.

Em relação à decomposição do tamanho médio $(\mathrm{x})$, note primeiramente que o tamanho de um estabelecimento está intimamente ligado ao setor de atividade. $^{7}$ Se $G:\{g=1, \ldots, n\}$ é uma partição do universo de firmas no setor formal - formada, por exemplo, por setores de atividade - o tamanho médio

\footnotetext{
${ }^{7}$ A conexão entre tamanho e setor de atividade vem da hipótese de que cada setor tem uma tecnologia específica de produção, que por sua vez define sua respectiva escala ótima.
} 
dessas empresas em um instante de tempo i pode ser escrito como uma média ponderada entre os tamanhos médios em diversos setores, ou seja:

$$
x_{i}=\sum_{g} w_{g i} \cdot x_{g i}
$$

onde $x_{g i}$ é o tamanho médio das firmas no g-ésimo grupo naquele instante de tempo e $w_{g i}$ é a proporção de firmas naquele grupo naquele instante de tempo. Dessa forma, podemos investigar em que medida a variação observada no tamanho médio dos estabelecimentos formais foi induzida por uma mudança na composição desse universo, no que tange ao setor de atividade. Por exemplo, é sabido que estabelecimentos industriais tendem a ser maiores do que os de comércio. Sendo assim, uma redistribuição dos estabelecimentos nos quais se aumenta a concentração da indústria pode induzir um aumento do tamanho médio sem que este aumente em cada um dos grupos considerados.

Seguindo a notação já estabelecida previamente (e novamente fixando $\alpha=$ $1 / 2)$, temos a seguinte igualdade para a variação do tamanho médio dos estabelecimentos entre o primeiro e o último ano de um dado subperíodo:

$$
\begin{aligned}
& x_{1}-x_{0}=\sum_{g} w_{g, 1} x_{g, 1}-\sum_{g} w_{g, 0} x_{g, 0} \\
& x_{1}-x_{0}=\sum_{g} w_{g, m}\left(x_{g, 1}-x_{g, 0}\right)+\sum_{g} x_{g, m}\left(w_{g, 1}-w_{g, 0}\right)
\end{aligned}
$$

onde, o primeiro termo à esquerda da equação (2) reflete o efeito de variações no tamanho médio das firmas no interior dos grupos - "efeito escala (setorial)" - e o segundo é o "efeito composição". O primeiro nos informa a contribuição da variação do emprego médio de cada setor " $g$ " (mantido fixo a participação relativa média entre os dois anos considerados), enquanto o segundo informa a contribuição da variação da participação relativa de cada setor no total de estabelecimentos (mantido fixo o emprego médio do setor).

\subsection{Resultados}

Na Tabela 1 reportamos a variação total do emprego formal (reportada na penúltima coluna), bem como a parcela referente a cada um dos dois componentes tratados na equação (1), para os subperíodos que apresentam as maiores diferenças na dinâmica do emprego formal, quais sejam, 1995-1999 e 2003-

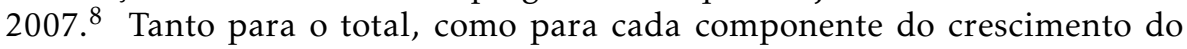
emprego, reportamos o valor absoluto em número de postos de trabalho $(\Delta)$, assim como uma taxa de crescimento em relação à média do emprego entre os períodos " 0 " e " 1 ". 9 .

A decomposição para os subperíodos mencionados mostra que a melhora na performance do emprego formal, de 2003-2007 em relação a 1995-1999,

\footnotetext{
${ }^{8}$ Doravante sempre constataremos a análise desses dois subperíodos.

${ }^{9}$ Dessa forma os números da última coluna, por exemplo, são dados por: $\left(x_{1} \cdot n_{1}-x_{0} \cdot n_{0}\right) /\left(x_{m}\right.$. $\left.n_{m}\right)$. A média do emprego no denominador torna-se necessária pelas ponderações dos componentes serem baseadas também em médias ( $\mathrm{nm}$ e xm respectivamente). Assim, a taxa da última coluna pode ser facilmente decomposta em taxa do crescimento do tamanho médio e do número médio de estabelecimentos: $\left[n_{m} \cdot\left(x_{1}-x_{0}\right)\right] /\left(n_{m} \cdot x_{m}\right)+\left[x_{m} \cdot\left(n_{1}-n_{0}\right)\right] /\left(n_{m} \cdot x_{m}\right)$. Esses dois componentes correpondem às taxas da segunda e quarta colunas, respectivamente.
} 
Tabela 1: O crescimento do emprego formal e de seus componentes (em milhares)

\begin{tabular}{|c|c|c|c|c|c|c|}
\hline & \multicolumn{2}{|c|}{$\begin{array}{c}\text { efeito tamanho médio } \\
n_{m} \cdot\left(x_{1}-x_{0}\right)\end{array}$} & \multicolumn{2}{|c|}{$\begin{array}{l}\text { efeito quantidade } \\
\left(n_{1}-n_{0}\right) \cdot x_{m}\end{array}$} & \multicolumn{2}{|c|}{$\begin{array}{c}\text { Total } \\
\left(x_{1} \cdot n_{1}-x_{0} \cdot n_{0}\right)\end{array}$} \\
\hline & $\Delta$ & $\operatorname{taxa}(\%)$ & $\Delta$ & $\operatorname{taxa}(\%)$ & $\Delta$ & $\operatorname{taxa}(\%)$ \\
\hline 95-99 & $-3,105$ & $-12,8$ & 4,540 & 18,7 & 1,434 & 5,9 \\
\hline $03-07$ & 2,751 & 8,3 & 5,221 & 15,7 & 7,972 & 24,0 \\
\hline
\end{tabular}

Fonte: Elaboração Própria a partir dos microdados da RAIS.

deve-se quase que exclusivamente a uma mudança drástica no efeito do tamanho médio dos estabelecimentos. Enquanto a taxa de crescimento do emprego devido ao efeito quantidade de estabelecimentos difere apenas em três pontos percentuais (p.p.) entre os dois subperíodos, a taxa de crescimento do emprego devido à variação do tamanho médio passa de $-12,8 \%$ para $+8,3 \%$, diferença de mais de 20 p.p.

Ou seja, a evolução do tamanho médio dos estabelecimentos não apenas parou de afetar negativamente a variação do emprego formal, como passou a contribuir para o seu aumento no último subperíodo analisado. Essa constatação torna lícito inferir que foi o crescimento médio do tamanho das firmas, mais do que o ritmo de aumento do número de firmas, o responsável pela mudança no padrão de expansão do emprego formal entre os dois subperíodos. Entretanto, é bom destacar que o efeito quantidade foi o mais importante para explicar o montante de postos gerados em ambos os subperíodos.

A despeito de alguns estudos mostrarem uma distinção na dinâmica do mercado de trabalho entre áreas metropolitanas e não-metropolitanas, a predominância do efeito do tamanho médio é comum as duas áreas como pode ser visto na Tabela A.1. do apêndice.

A Tabela 2 nos mostra os resultados da decomposição do tamanho médio em efeito escala e efeito composição, baseada na equação (2) de forma análoga aos reportados na Tabela $1 .{ }^{10}$ Observamos que o efeito escala é o grande responsável pela mudança de tendência observada no tamanho médio entre os dois subperíodos. Entre 1995 e 1999 esse efeito afetava negativamente o emprego formal, que encolheria em $11,5 \%$ caso o efeito composição fosse nulo. Já, no segundo subperíodo, o crescimento do emprego formal seria de 10,5\% caso o efeito escala fosse o único determinante do emprego formal.

Na Tabela A.2 do Apêndice reproduzimos a Tabela acima aberta por setor de atividade das firmas. De forma geral, o efeito escala, que era negativo, passou a atuar positivamente no segundo subperíodo para quase todos os setores de atividade. ${ }^{11}$ Portanto, até o momento temos evidências de que houve

\footnotetext{
${ }^{10}$ Multiplicamos os efeitos escala e composição pelo número médio de estabelecimentos (nm) entre os anos do subperíodo que está sendo analisado. Assim, temos o impacto do efeito escala e composição em termos de nível médio de emprego. Assim, pode-se notar que a variação total é exatamente igual ao efeito tamanho médio, observado na Tabela 1, para cada subperíodo.

${ }^{11}$ Essa reversão é registrada para 22 dos 25 setores investigados, e de magnitude superior a 15 p.p. em 21 setores. Por exemplo, observamos que todos os setores da indústria registraram uma diminuição no tamanho médio no primeiro subperíodo. Esta redução, ocorrida na década de 90 e já apontada por Saboia (2000), foi ligada ao processo de downsizing (enxugamento das empresas). Este processo ocorreu principalmente pelo aumento da terceirização, mudança tecnológica mais intensiva em capital (devido à abertura comercial) e pela maior utilização de mão-de-obra qualificada (em detrimento da não-qualificada). Isso fez com que a produtividade marginal do
} 
Tabela 2: Decomposição da Variação do Tamanho Médio em Efeito Escala e Efeito Composição

\begin{tabular}{|c|c|c|c|c|c|c|}
\hline & \multicolumn{2}{|c|}{$\begin{array}{c}\text { efeito escala } \\
n_{m} \cdot w_{m} \cdot\left(x_{1}-x_{0}\right)\end{array}$} & \multicolumn{2}{|c|}{$\begin{array}{c}\text { efeito composição } \\
n_{m} \cdot x_{m} \cdot\left(w_{1}-w_{0}\right)\end{array}$} & \multicolumn{2}{|c|}{$\begin{array}{c}\text { Total } \\
n_{m} \cdot\left(x_{1}-x_{0}\right)\end{array}$} \\
\hline & $\Delta$ & $\operatorname{taxa}(\%)$ & $\Delta$ & taxa $(\%)$ & $\Delta$ & $\operatorname{taxa}(\%)$ \\
\hline 95-99 & $-2,798$ & $-11,5$ & -308 & $-1,3$ & $-3,105$ & $-12,8$ \\
\hline 03-07 & 3,497 & 10,5 & -747 & $-2,2$ & 2,751 & 8,3 \\
\hline
\end{tabular}

Fonte: Elaboração Própria a partir dos microdados da RAIS.

de fato uma reversão na tendência do tamanho médio das firmas, sem que isso tenha sido mascarado por um efeito composição. A partir desse ponto, não nos parece óbvio como proceder para investigar o que pode ter levado o tamanho médio crescer. Sendo assim, recorreremos à teoria econômica para buscar um mecanismo de determinação do nível de emprego pelas firmas que permita investigar empiricamente a evolução dos seus determinantes.

\section{Efeito seleção ou efeito escala: argumentos teóricos}

Nessa seção recorreremos à teoria econômica para aprofundar nossa análise sobre os determinantes do tamanho médio de um estabelecimento. Assumiremos que o arcabouço desenvolvido por Pakes \& Ericson (1998), que remonta ao artigo seminal de Jovanovic (1982), cumpre esse papel de forma satisfatória, dado que algumas implicações desse arcabouço foram testadas com sucesso por uma diversidade de trabalhos empíricos. ${ }^{12}$ Sendo assim, faremos uma breve exposição da teoria, focando no que ela tem a dizer sobre as decisões da firma referente a tamanho, para, em seguida, checar em que medida o arcabouço nos ajuda a interpretar os fatos mostrados acima.

\subsection{Hipóteses}

A principal hipótese do modelo de Pakes \& Ericson (1998) é que a função de produção de uma firma " $i$ ", e, portanto também o seu lucro, são afetados por um termo $u_{i t}$, que por sua vez é influenciado pelo grau de eficiência inato de cada firma $\left(\theta_{i}\right)$. As hipóteses adicionais necessárias para a formulação da decisão da firma são: i) o trabalho é o único insumo produtivo e ii) as firmas são tomadoras de salário $\left(w_{t}\right)$. Dessa forma, o lucro corrente da firma $\left(\pi_{i t}\right)$ pode ser descrito da seguinte maneira:

$$
\pi_{i} t=p_{t} \cdot u_{i t}\left(\theta_{i}\right) \cdot f\left(\ell_{i t}\right)-w_{t} \cdot \ell_{i t},
$$

onde $p_{t}$ corresponde ao preço unitário do único bem produzido na economia. Por um lado supõe-se que as firmas conhecem toda a trajetória de $p_{t}$, que será

capital e do trabalho aumentassem (que é justamente o objetivo do processo de downsizing). Isso ocorreu motivado por condições macroeconômicas internas e externas desfavoráveis às firmas principalmente pequenas - como, por exemplo, regime cambial fixo e as diversas crises econômicas mundiais que ocorreram no período. Em contraste, observamos um cenário macroeconômico mais favorável no segundo período, pela estabilidade do cenário externo e política cambial mais flexível e, portanto, favorável às pequenas empresas. Por isso, no segundo subperíodo, o setor industrial apresentou um aumento no tamanho médio. Na maioria dos casos, os efeitos escala e composição passam a influenciar positivamente.

${ }^{12}$ Ver por exemplo as resenhas de Sutton (1997), Caves (1998) e Bartelsman \& Doms (2000). 
aqui denotada por $p$, mas por outro lado supõe-se que a firma não sabe o valor de $u_{i t}$ a que ela estará submetida ao longo de $t$. Esse valor será revelado para firma apenas no final do período. O que se sabe sobre $u_{i t}$ no início do período é o seguinte:

i) $u_{i t}=\theta_{i}+v_{i t}$

ii) $v_{i t} \sim N\left(0, \sigma^{2}\right)$ iid

Ou seja, $u_{i t}$ pode ser interpretado como um sinal que a firma recebe sobre o componente inato do seu grau de eficiência. A coincidência entre essas duas variáveis é impedida por um ruído $\left(v_{i t}\right)$, que oscila em torno de zero.

Dada a ausência de custos de ajustamento ou qualquer outro fator que torne a escolha de $\ell_{i t}$ uma decisão intertemporal, a firma toma essa decisão maximizando o lucro corrente. A fim de garantir solução única, o autor impõe um formato côncavo para a função $f(\cdot)$.

Com relação à decisão do nível ótimo de trabalho $\left(\ell_{i t}^{*}\right)$, essa decisão será baseada na expectativa que a firma tem sobre $u_{i} t$. A partir das hipóteses i) e ii) acima, é fácil deduzir que $E\left[u_{i t}\right]=\theta_{i}$, quando a firma conhece $\theta$ i. Porém, a firma também não sabe o verdadeiro valor de $\theta_{i}$. Sabe-se apenas que a distribuição dessa variável aleatória entre as firmas também é normal com média $\theta^{0}$ e variância $\sigma_{0}^{2} \cdot{ }^{13}$ A firma usa a informação dos sinais que ela recebe para inferir de forma bayesiana essa variável aleatória, de forma que a firma escolhe a quantidade a ser produzida maximizando a seguinte definição de lucro esperado:

$$
\max _{\ell_{i t}} E\left[\pi_{i t}\right]=\max _{\ell i t} p_{t} \cdot E\left[\theta \mid u_{i 1} \ldots u_{i t-1}\right] \cdot f\left(\ell_{i t}\right)-w_{t} \cdot \ell_{i t} .
$$

Outra decisão importante tomada pela firma é a permanência no mercado. No início de cada instante a firma decide fechar (pra sempre) ou permanecer mais um período. Note que essa decisão é de caráter intertemporal, dado que o que for decidido no instante $t$ terá impacto sobre as decisões a serem tomadas em $t+1$.

\subsection{Resultados}

Jovanovic (1982) e, por consequência Pakes \& Ericson (1998), derivam uma série de resultados que são explorados pela literatura empírica sobre dinâmica de firmas. Aqui, vamos nos concentrar naqueles mais diretamente relacionados com o objetivo desse artigo, quais sejam, as decisões sobre permanência no mercado e nível de emprego.

Em relação à decisão do nível ótimo de emprego, temos a seguinte relação:

1. $\ell_{i t}^{*}=\ell\left(p_{t} \cdot E\left[\theta \mid u_{i 1} \ldots u_{i t-1}\right] / w\right)$, de tal forma que essa função é crescente em $E\left[\theta \mid u_{i 1} \ldots u_{i t-1}\right]$. Logo o formato da distribuição cross-section de $\ell_{i t}^{*}$ segue o formato da distribuição de $E\left[\theta \mid u_{i 1} \ldots u_{i t-1}\right]$.

Por fim, em relação à tomada de decisão sobre a permanência da firma no mercado temos o seguinte resultado:

\footnotetext{
${ }^{13}$ Como consequência desse fato, todas as firmas tem a mesma expectativa em relação ao seu grau de ineficiência no início do primeiro período de atividade. Ou seja, $\theta^{0}$ é o valor da expectativa média das firmas nascentes.
} 


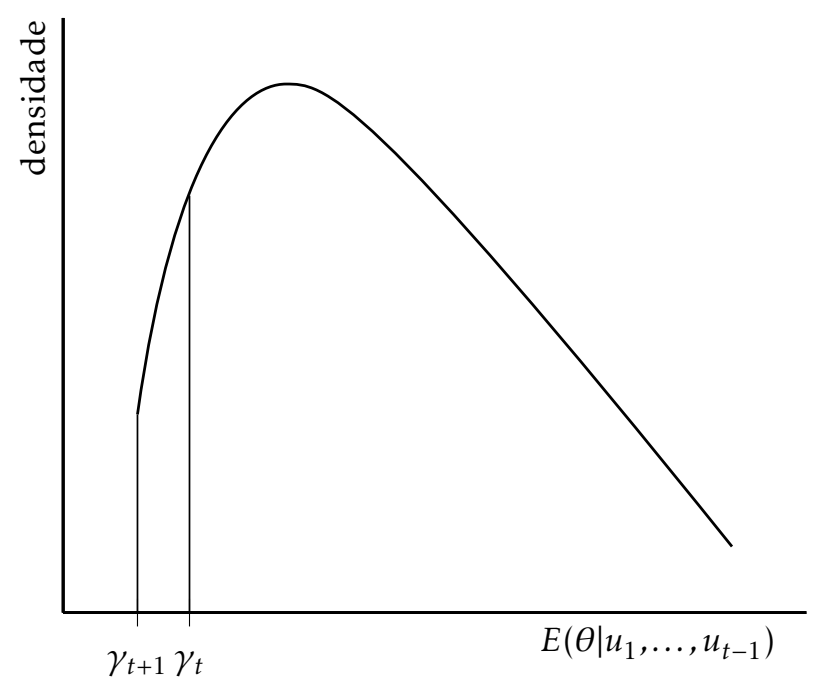

Figura 5: Deslocamento Hipotético do limite $\gamma_{t}\left(n ; p_{t}\right)$

2. Para cada idade da firma $(n)$, e cada instante do tempo existe um nível limite de $E\left[\theta \mid u_{i 1} \ldots u_{i t-1}\right]$ que define a permanência da firma. Chamaremos esse limite de $\gamma_{t}\left(n ; p_{t}\right)$. Quando $E\left[\theta \mid u_{i 1} \ldots u_{i t-1}\right]$ se encontra abaixo desse limite a firma decide encerrar suas atividades, e permanece no mercado caso contrário.

Esses resultados têm implicações importantes para nossa análise da evolução do emprego formal. Em primeiro lugar as distribuições crosssection tanto de $E\left[\theta \mid u_{i 1} \ldots u_{i t-1}\right]$ como do tamanho das firmas sobreviventes são truncadas à esquerda. Em decorrência disso a média do tamanho das sobreviventes pode ser afetada por alterações no valor de $\gamma_{t}$, sem que nenhuma firma altere seu nível de emprego. A Figura 5 ilustra uma mudança hipotética na distribuição de eficiência (ou tamanho) derivada de uma simples redução deste limite, de $\gamma_{t}$ para $\gamma_{t+1}$. Isso faz com que parte das firmas que morreriam antes passe a sobreviver. Essas firmas tendem a ter níveis de eficiência e tamanho menor do que aquelas que sobrevivem em ambos os cenários, fazendo com que o nível médio do emprego da economia caia. Um exemplo que poderia causar a queda do $\gamma$ seria a instituição do SIMPLES, que permitiu que firmas pequenas (e de baixo nível de eficiência) que antes morreriam, passam a sobreviver ao usufruir das melhores condições que este programa oferece (redução da alíquota tributária e da burocracia). Chamaremos o componente da evolução do emprego médio atribuído a mudanças em $\gamma$ de efeito seleção, enquanto o componente atribuído à evolução de $\ell^{*}$ de efeito escala da firma. Assim, existe a necessidade de investigar em que medida esse fenômeno de seleção amostral vem contribuindo para a evolução do emprego formal, distinguindo-o do efeito escala. A identificação da contribuição isolada de cada um desses dois efeitos requer a exploração de outros resultados do modelo que seguem abaixo.

3. Em um dado intervalo de tempo $(\Delta t)$ os valores limites $\gamma_{t}\left(n ; p_{t}\right)$ de duas faixas etárias distintas não podem se mover em direções opostas, ou seja, 
o sinal de $\left(\gamma / p_{t}\right)$ deve ser o mesmo para valores de $\mathrm{n}$ diferentes.

4. Os valores de $\theta^{0}$ e $\sigma_{0}^{2}$ vigentes em um dado instante $t$ influencia apenas o nível de emprego dos estabelecimentos criados nesse mesmo instante do tempo. Ou seja, caso haja uma mudança exógena de $\theta^{0}$ e $\sigma_{0}^{2}$, isso não afeta $E\left[\theta \mid u_{i 1} \ldots u_{i t-1}\right]$ da firma que já tem $t-1>2$ anos, e portanto não afeta o seu $\ell_{i t}^{*}$. Ou seja, ao contrário do que ocorre com $\gamma_{t}, \ell_{i t}^{*}$ pode não variar homogeneamente entre faixas etárias.

5. Para uma dada coorte a influência de $\theta^{0}$ em $E\left[\theta \mid u_{i 1} \ldots u_{i t-1}\right]$ diminui com a idade. Logo, o mesmo vale para o nível de emprego.

Assim, na próxima seção faremos algumas inferências sobre como evoluem $\gamma_{t}(n ; p)$ e $\ell^{*}$ por faixa etária, tendo em mente os resultados acima expostos. ${ }^{14}$

\section{Efeito seleção ou efeito escala: resultados empíricos}

A seção anterior motivou a necessidade de investigar em que medida esse fenômeno de seleção amostral vem contribuindo para a evolução do emprego formal. Como $\gamma$ varia com a idade, torna-se necessário condicionarmos essa análise na faixa etária dos estabelecimentos. A fim de investigar hipóteses desse tipo, analisaremos os resultados da Tabela 3 sobre a frequência por tamanho do estabelecimento em cada faixa etária. Na última linha da Tabela apresentamos também o emprego médio do primeiro e segundo subperíodo por faixa etária. A idade considerada foi a do ano mediano de cada subperíodo, ou seja, 1997 e 2005. Nas demais linhas da Tabela cada número diz respeito ao percentual de estabelecimentos com tamanho médio indicado na respectiva linha no total de estabelecimentos da faixa etária indicada na coluna.

O primeiro resultado que nos chama a atenção é a clara distinção na evolução do emprego médio por faixa etária. Enquanto nas duas primeiras faixas etárias o emprego cresce, de 1997 para 2005, na faixa dos estabelecimentos mais antigos o emprego médio registra uma queda. Os resultados referentes à participação dos pequenos estabelecimentos vão no mesmo sentido. Nas duas primeiras faixas etárias há uma queda do percentual de estabelecimentos com menos que cinco empregados entre o primeiro e o segundo sub-período. Já na faixa etária mais avançada ocorre o contrário.

Caso esses movimentos fossem explicados exclusivamente por variações nos respectivos valores de censura a partir do qual os estabelecimentos decidem encerrar suas atividades, deveríamos ter, de acordo com os resultados 1) e 2), um aumento desse valor de corte para os novos estabelecimentos e uma diminuição do valor de corte na faixa etária mais avançada. No entanto, esta interpretação vai de encontro ao resultado 3 ).

Uma hipótese que nos parece compatível com esses resultados é que pode ter havido uma diminuição generalizada nos respectivos valores de corte por faixa etária, mas esse movimento pode ter ocorrido simultaneamente com um aumento do nível de emprego restrito as firmas mais jovens, como mostrado na Figura 2 e justificado pela hipótese 4). Ou seja, os resultados apontam na

\footnotetext{
${ }^{14}$ Nossa pretensão aqui não é inferir os parâmetros estruturais do modelo exposto, mas apenas lançar mão de seus resultados a fim de verificar a possibilidade da existência de um efeito seleção das firmas.
} 
Tabela 3: Frequência por classe de tamanho em cada faixa etária (em percentagem)

\begin{tabular}{|c|c|c|c|c|c|c|}
\hline \multirow[b]{3}{*}{ tamanho } & \multicolumn{6}{|c|}{ Faixa etária } \\
\hline & \multicolumn{3}{|c|}{1997} & \multicolumn{3}{|c|}{2005} \\
\hline & 1 ano & 2-4 anos & $5+$ anos & 1 ano & 2-4 anos & $5+$ anos \\
\hline 1 a 4 & 77,88 & 75,10 & 55,80 & 76,63 & 70,87 & 58,79 \\
\hline 5 a 9 & 12,42 & 13,58 & 19,49 & 12,88 & 15,43 & 19,18 \\
\hline 10 a 19 & 5,74 & 6,42 & 11,58 & 6,02 & 7,71 & 11,06 \\
\hline 20 a 49 & 2,64 & 3,29 & 7,49 & 3,10 & 4,00 & 6,58 \\
\hline 50 a 99 & 0,78 & 0,89 & 2,65 & 0,80 & 1,16 & 2,10 \\
\hline 100 a 249 & 0,38 & 0,51 & 1,80 & 0,39 & 0,57 & 1,32 \\
\hline 250 a 499 & 0,11 & 0,13 & 0,69 & 0,12 & 0,16 & 0,52 \\
\hline 500 a 999 & 0,04 & 0,05 & 0,32 & 0,04 & 0,06 & 0,27 \\
\hline 1000 a 4999 & 0,02 & 0,02 & 0,17 & 0,02 & 0,04 & 0,15 \\
\hline $5000+$ & 0,00 & 0,00 & 0,02 & 0,00 & 0,00 & 0,02 \\
\hline $\begin{array}{l}\text { Média do } \\
\text { Emprego }\end{array}$ & 6,02 & 8,08 & 22,04 & 6,47 & 8,62 & 18,68 \\
\hline
\end{tabular}

direção de um aumento do nível de emprego ótimo restrito às firmas jovens. Exemplos de aumento de $\theta^{0}$ podem estar relacionados a mudanças no ambiente econômico, tal como redução de incertezas devido a um ambiente institucional e regime cambial mais "seguros" e avanços tecnológicos no segundo subperíodo. Com essa menor incerteza, as firmas acabam tendo um investimento inicial maior e nascem, em média, maiores, em relação ao primeiro subperíodo, que apresentou condições macroeconômicas internas e externas desfavoráveis às firmas - principalmente para as pequenas. ${ }^{15}$

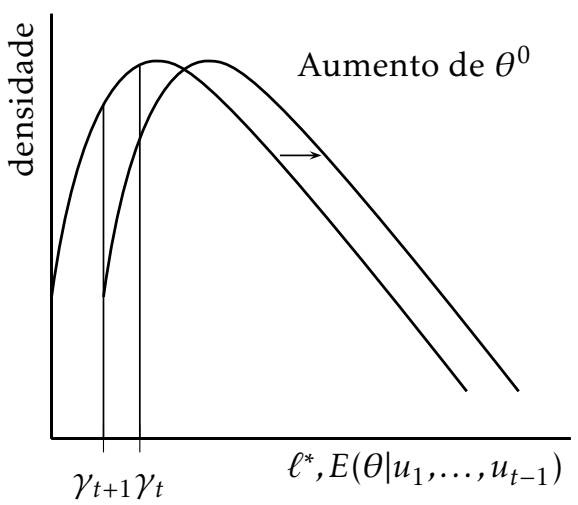

Faixas etárias 1 e 2-4 anos

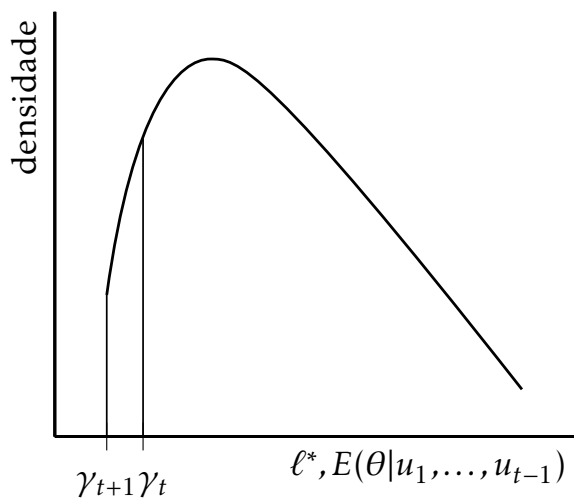

Faixas etárias $5+$ anos

Figura 6: Deslocamento Hipotético do limite $\gamma_{t}(\mathrm{n} ; \mathrm{pt})$ e $\theta^{0}$ por faixas etárias

A Tabela 4 mostra evidências compatíveis com a interpretação de que de fato houve uma diminuição em $\gamma$. Nas duas primeiras colunas temos a taxa

\footnotetext{
${ }^{15}$ Esse ponto já foi apontado na nota 11.
} 
de sobrevivência média de cada um dos respectivos subperíodos analisados. Embora a taxa de sobrevivência tenha aumentado para todas as classes de tamanho, a terceira coluna da Tabela explicita que quanto menor o tamanho, maior foi o aumento na taxa de sobrevivência. Esse fato corrobora nossa hipótese de que a seleção está sendo mais tênue (deslocamento para esquerda do $\gamma$ ), e empresas menores passam a ter maiores chances de sobreviver no segundo sub-período.

Tabela 4: Probabilidade de Sobrevivência por classe de tamanho (em \%)

\begin{tabular}{lllc}
\hline tamanho & $95-99$ & $03-07$ & $\Delta$ \\
\hline 1 a 4 & 78,19 & 80,81 & 3,35 \\
5 a 9 & 90,90 & 93,68 & 3,06 \\
10 a 19 & 92,44 & 95,14 & 2,91 \\
20 a 49 & 93,69 & 96,09 & 2,57 \\
50 a 99 & 94,23 & 96,55 & 2,46 \\
100 a 249 & 94,83 & 96,90 & 2,19 \\
250 a 499 & 95,52 & 97,21 & 1,76 \\
500 a 999 & 96,10 & 97,65 & 1,61 \\
1000 a 4999 & 96,32 & 97,68 & 1,41 \\
5000 + & 96,21 & 97,48 & 1,32 \\
\hline Fonte: Elaboração Própria a partir dos \\
microdados da RAIS.
\end{tabular}

Por fim, o resultado (5) justifica, o fato de o movimento em $\gamma$ predominar na faixa etária mais avançada enquanto o movimento em $\theta^{0}$ predomina nas faixas mais jovens. Ou seja, as firmas passaram a nascer com um emprego médio maior do primeiro para o segundo subperíodo, dadas as condições macroeconômicas mais favoráveis, já citadas.

\section{Conclusão}

A evolução do emprego formal mostra tendências bem distintas entre as décadas de 1990 e 2000, passando a apresentar um ritmo de crescimento bem mais acentuado na última década. Procuramos mostrar quais os determinantes do emprego formal que poderiam ser apontados como responsáveis por essa mudança de tendência. Ao decompor essa evolução entre número de estabelecimentos e tamanho médio dos mesmos, é nítido que esse último componente é o grande responsável pela mudança de ritmo sob investigação. Esse panorama inclusive é mantido quando replicamos esse procedimento para recortes regionais.

Investigações mais detalhadas descartam a hipótese de uma mudança na composição onde o peso de setores com maior escala de produção teria aumentado. Por outro lado há evidência compatível com a hipótese de que houve aumento generalizado no tamanho médio dos estabelecimentos recémcriados. Esse efeito teria predominado sobre o aumento detectado na probabilidade de sobrevivência de estabelecimentos menores. 
Seria difícil apontar com precisão os fatores institucionais e de políticas públicas que podem estar por trás desses resultados, porém, vale mencionar algumas medidas recentes compatíveis com esses resultados, adicionalmente à redução de incertezas no ambiente macroeconômico vivido na atual década. Do ponto de vista do aumento do tamanho médio dos estabelecimentos recém-criados, valeria a pena investigar o papel dos novos procedimentos de abertura de firmas implementados tanto pela Receita Federal como por órgãos locais tais como juntas comerciais e secretarias de fazenda estaduais e municipais. Essas medidas podem ter reduzido bastante o custo de abrir uma firma permitindo que uma maior parte do capital inicial seja alocada a produção. Outra medida com potencial para afetar tanto o tamanho das firmas novas como também a sobrevivência das menores é a implementação de sistemas tributários especiais para pequenas empresas, tais como o SIMPLES Federal de 1997 (Corseuil \& Moura 2009).

\section{Apêndice A}

Tabela A.1: O crescimento do emprego formal e de seus componentes (em milhares)

\begin{tabular}{|c|c|c|c|c|c|c|}
\hline \multicolumn{7}{|c|}{ Áreas não metropolitanas } \\
\hline & \multicolumn{2}{|c|}{$\begin{array}{c}\text { efeito tamanho médio } \\
n_{m} \cdot\left(x_{1}-x_{0}\right)\end{array}$} & \multicolumn{2}{|c|}{$\begin{array}{l}\text { efeito quantidade } \\
\left(n_{1}-n_{0}\right) \cdot x_{m}\end{array}$} & \multicolumn{2}{|c|}{$\begin{array}{c}\text { Total } \\
\left(x_{1} \cdot n_{1}-x_{0} \cdot n_{0}\right) \\
\end{array}$} \\
\hline & $\Delta$ & taxa & $\Delta$ & $\operatorname{taxa}$ & $\Delta$ & $\operatorname{taxa}(\%)$ \\
\hline $95-99$ & $-1,402$ & $-12,1$ & 2,662 & 23,0 & 1,261 & 10,9 \\
\hline $03-07$ & 1,403 & 8,1 & 2,986 & 17,3 & 4,388 & 25,4 \\
\hline \multicolumn{7}{|c|}{ Áreas metropolitanas } \\
\hline & \multicolumn{2}{|c|}{$\begin{array}{c}\text { efeito tamanho médio } \\
n_{m} \cdot\left(x_{1}-x_{0}\right)\end{array}$} & \multicolumn{2}{|c|}{$\begin{array}{l}\text { efeito quantidade } \\
\quad\left(n_{1}-n_{0}\right) \cdot x_{m}\end{array}$} & \multicolumn{2}{|c|}{$\begin{array}{c}\text { Total } \\
\left(x_{1} \cdot n_{1}-x_{0} \cdot n_{0}\right) \\
\end{array}$} \\
\hline & $\Delta$ & taxa & $\Delta$ & taxa & $\Delta$ & $\operatorname{taxa}(\%)$ \\
\hline $95-99$ & $-1,437$ & $-11,3$ & 1,611 & 12,7 & $17 \overline{3}$ & 1,4 \\
\hline $03-07$ & 1,482 & 9,3 & 2,102 & 13,1 & 3,583 & 22,4 \\
\hline
\end{tabular}

Fonte: Elaboração Própria a partir dos microdados da RAIS. 


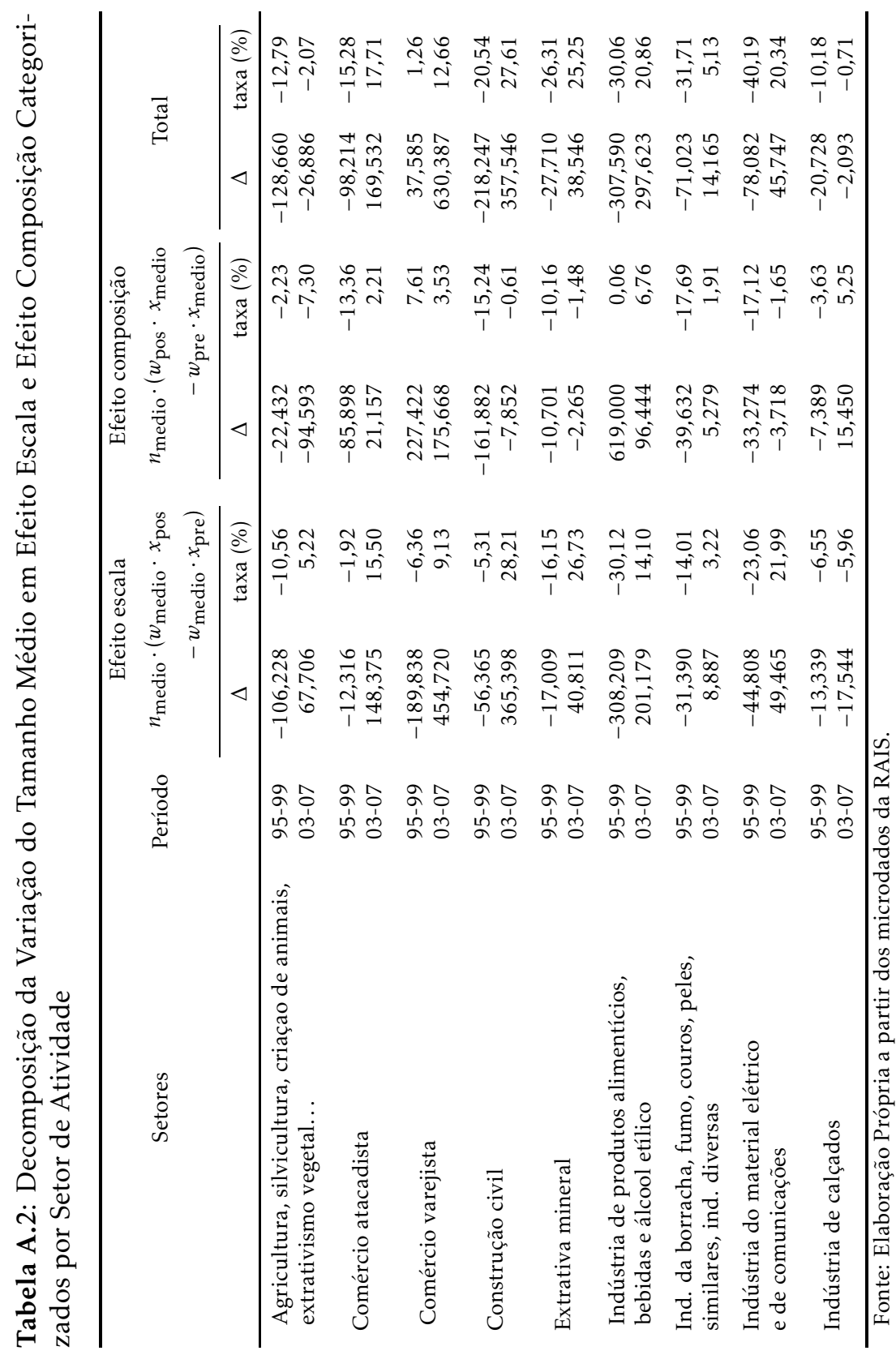




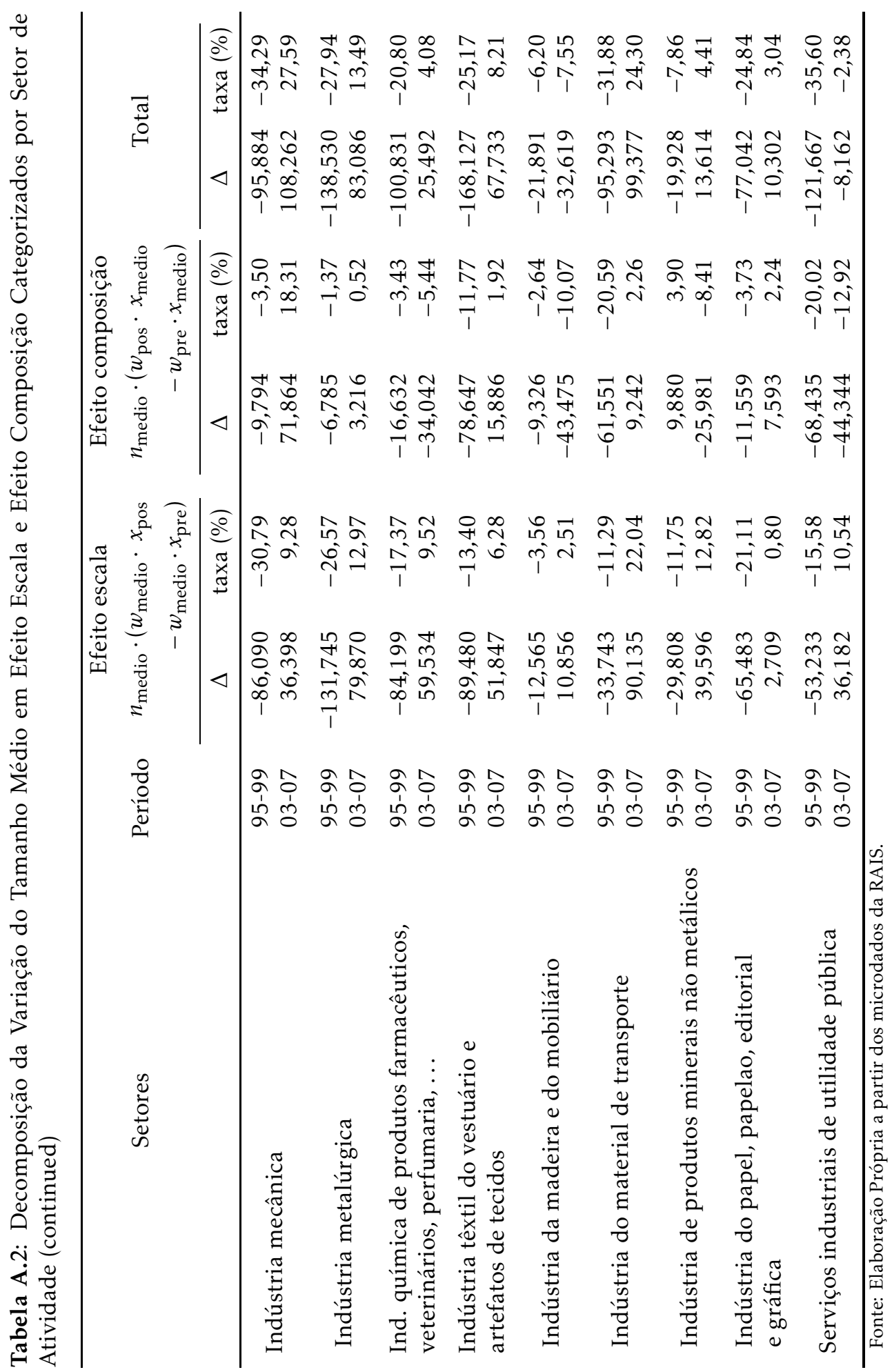




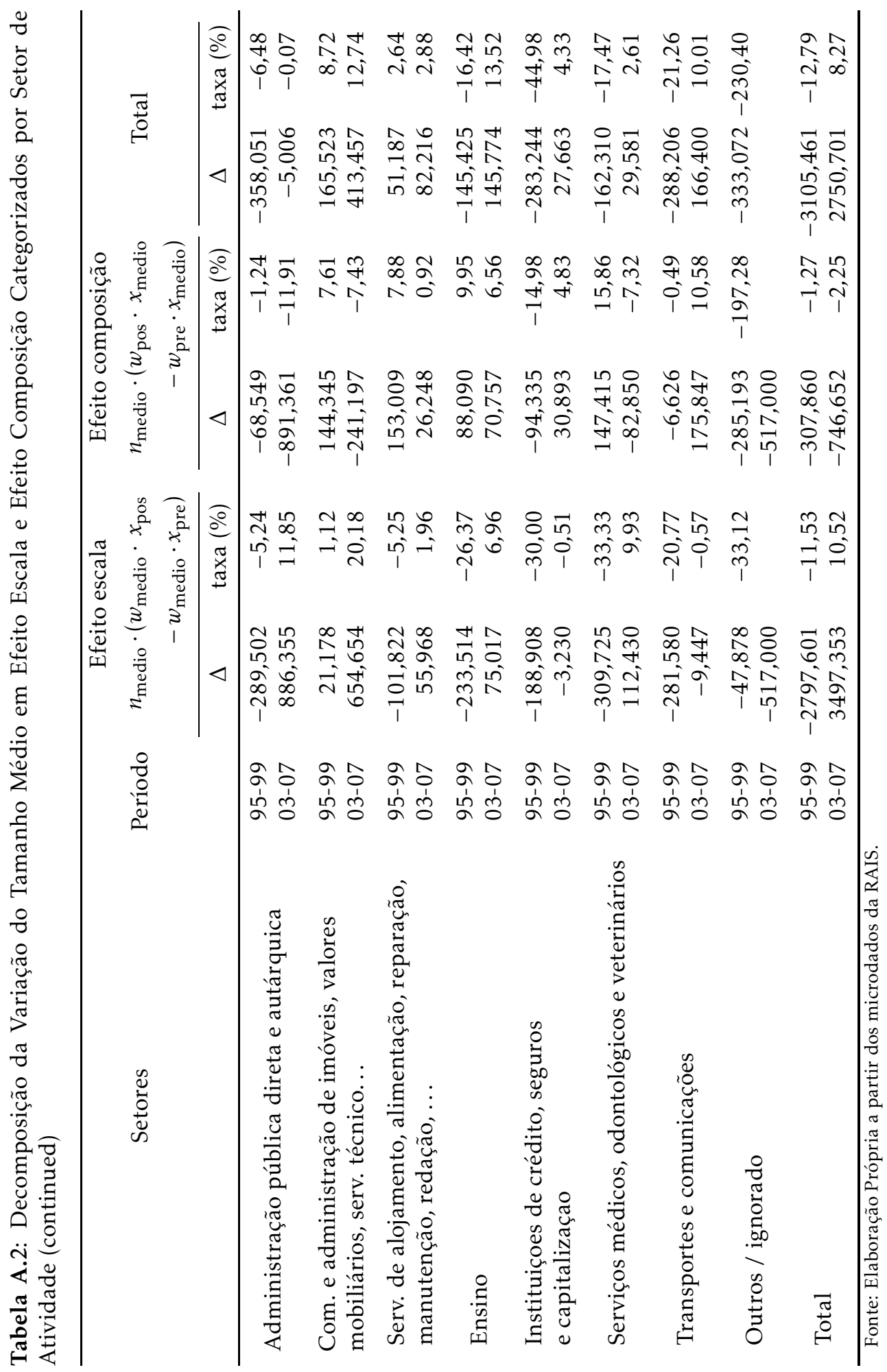




\section{Referências Bibliográficas}

Bartelsman, E. \& Doms, M. (2000), 'Understanding productivity: Lessons from longitudinal microdata', Journal of Economic Literature 38, 569-594.

Cavaleri, R. (2008), A expansão da ocupação em 2007, in 'Mercado de Trabalho - Conjuntura e Análise', Ipea, Rio de Janeiro.

Caves, R. (1998), 'Industrial organization and new findings on the turnover and mobility of firms', Journal of Economic Literature 36, 1947-1982.

Chahad, J. (2006), O emprego formal no Brasil entre 1992-2006: comportamento, tendências atuais e causas explicativas, in 'O Mercado de Trabalho Formal no Brasil', J. Macambina, (Ed). Imprensa Universitária UFCE, Fortaleza.

Corseuil, C. \& Moura, R. (2009), O SIMPLES federal e a Geração de Empregos na Indústria.

Jovanovic, B. (1982), 'Selection and the evolution of industry', Econometrica 50, 649-670.

Pakes, A. \& Ericson, R. (1998), 'Empirical implications of alternative models of firm dynamics', Journal of Economic Theory 79, 1-46.

Ramos, L. \& Ferreira, V. (2005), Padrões espacial e setorial da informalidade no Brasil: 1991-2003, Textos para discussão, IPEA.

Saboia, J. (2000), 'Desconcentração industrial no Brasil nos anos 90: um enfoque regional', Pesquisa e Planejamento Econômico 30, 69-116.

Simão, A. (2009), Fiscalização do trabalho e simplificação tributária no Brasil - análise de seus efeitos sobre o emprego formal no período 1999/2007, in 'Dissertação de Mestrado', UFRJ, Rio de Janeiro.

Sutton, J. (1997), 'Gibrat's legacy', Journal of Economic Literature 35, 40-59.

Tafner, P., ed. (2006), Brasil - O Estado de uma nação, IPEA. 